\title{
Multi-Domain Vehicle Dynamics Simulation in Dymola
}

\author{
Andreas Deuring $^{\mathrm{a}} \quad$ Johannes Gerl $^{\mathrm{a}} \quad$ Dr. Harald Wilhelm ${ }^{\mathrm{b}}$ \\ ${ }^{a}$ Modelon GmbH München \\ ${ }^{\mathrm{b}}$ Audi AG Ingolstadt \\ andreas.deuring@modelon.com johannes.gerl@modelon.com \\ harald.wilhelm@audi.de
}

\section{Abstract}

In future cars, battery electric and hybrid electric drives will increasingly appear. Subsystems like e.g. the steering system and the braking system will accordingly be based on electric power supply. This leads to new challenges as well as opportunities also in the field of vehicle dynamics and an increased need of multidomain simulation concepts that combine multibody-based vehicle dynamics models and models of the electric and control systems. This paper includes a simulation study of the Audi sports car e-tron with electric power steering system using the Vehicle Dynamics Library from Modelon AB Sweden to model chassis and suspensions and the Modelica Standard Library to model the electric power steering system. The steering system controller unit was modeled alternatively in the Modelica Standard Library and in Matlab Simulink. Dymola and Matlab Simulink have alternatively been used as simulation environments whereas a special focus was put on different ways to integrate these tools according to standard development processes in the automotive industry. Additionally, extensive validation work was invested to compare vehicle dynamics results generated with ADAMS/Car and the Vehicle Dynamics Library.

\section{Introduction}

As hybrid and electric cars can store a higher amount of electric energy and dispose of higher voltage levels, it stands to reason to base subsystems like the steering system fully on electric power supply.

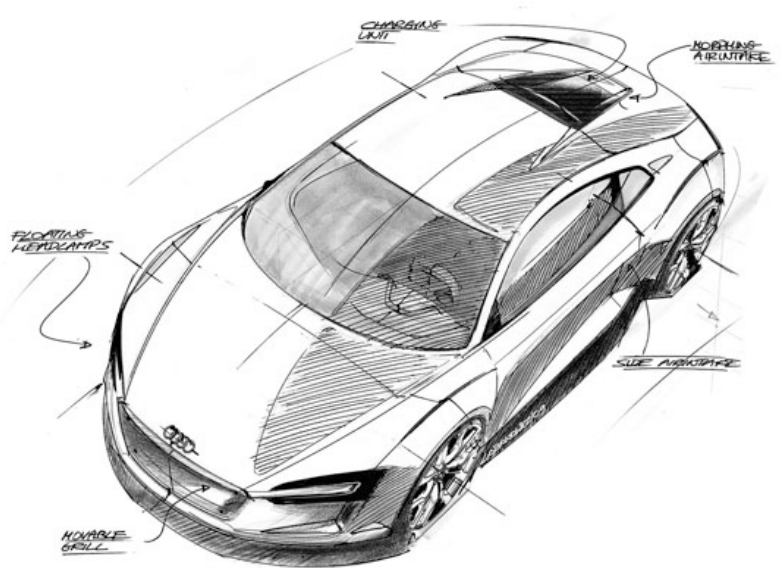

Fig. 1, Design Sketch of Electric Drive Sports

Car Audi e-tron, Picture: www.audi.de

Moreover electric drive systems offer the opportunity to give controlled input to the wheel torque in order to optimize the handling and the safety of the vehicle. For example concepts which provide a combination of standard propulsion technology on one axle and electric drives on the other, offer certain potential regarding handling behaviour, however, require high attention to ensure save driving in all conditions, e.g. during recuperation phases. As vehicle dynamics interfere with the dynamics of the electric systems and as an integrated control concept is required that includes vehicle dynamics and drive control systems, the usage of a multidomain simulation environment has obvious advantages compared to specialized tools with e.g. purely signal oriented or mechanical focus. In order to study the suitability of Dymola and the above named libraries, within this project a vehicle dynamics model of the Audi sports car e-tron was set-up and extensively verified and optimised towards an existing ADAMS/Car model. As an example for various electric systems, the electric steering sys- 
tem was added to the vehicle dynamics model. Finally, the controller was modelled both in Dymola and Matlab Simulink. The entire system model was simulated within Dymola (importing the controller model via Functional Mock-up Interface [2]) and Simulink (importing the Modelica based models via the standard Dymola - Simulink Interface).

\section{The Vehicle Dynamics Model Used}

The model of the vehicle dynamics in terms of the mechanical system was carried out using the Vehicle Dynamics Library (VDL) [1]. It contains fully detailed multibody models of the double wishbone front and rear suspensions of the car, whereas the single suspension links are interconnected with nonlinear bushing elements. In Fig. 2 the Dymola Model of the front suspension linkage subsystem is shown in detail. The models are based on VDL standard templates.

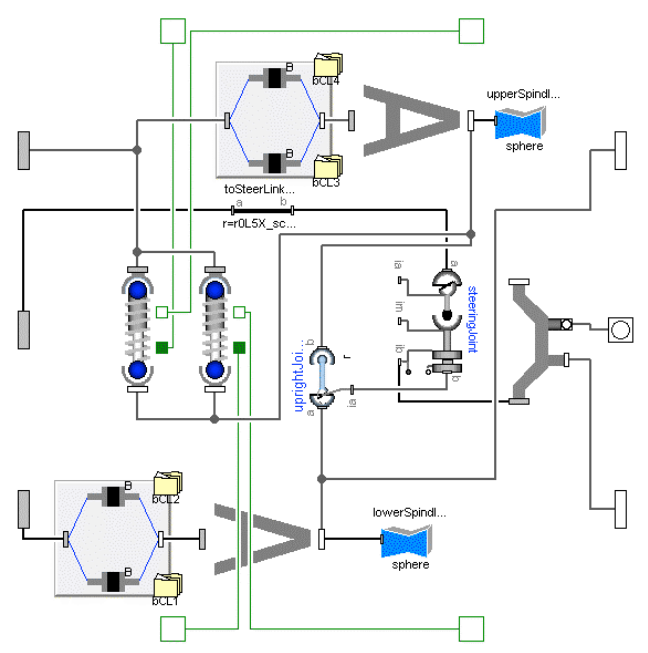

Fig. 2, VDL subsystem model of the double wishbone right linkage in Dymola and Vehicle Dynamics Lib

Fig. 3 gives a showcase overview of the full vehicle as it is graphically displayed in the animation tool of Dymola. The focus of the modelling work lied on the resolved rear and front suspensions. The car body and the subframes are modelled as rigid parts having six degrees of freedom each. The structure of the VDL-model was based on an already existing ADAMS/Car model with comparable complexity. The assembly of the VDL subsystems with the relevant multibody data like masses, inertias, geometry points, elasticities, damping, etc. was transferred and adopted from this ADMAS/Car model.

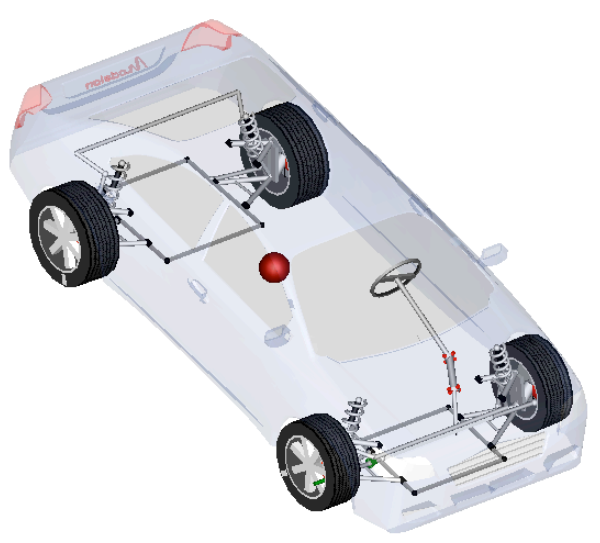

Fig. 3, Graphical animation of the full vehicle model in Dymola with focus on the resolved suspensions

However, the ADAMS model was not realised in any detail, as this was not in focus of the project. Differences in the simulation results aroused from certain elasticities of the suspension models that have not been taken into consideration in the VDL, due to the wish to work with standard templates.

\section{Comparison of the VDL and the ADAMS/Car model}

To compare and validate the VDL towards the ADAMS/Car model, experiments in the field of suspension kinematic and compliance analysis (K\&C) have been set up as well as fullvehicle handling experiments. For the K\&C tests, elasto-kinematic models have been created, optimised and then used in identical form in the full vehicle analysis. The results shown exemplarily in the following two figures contain a small cutout of the entire set of results that was achieved. 


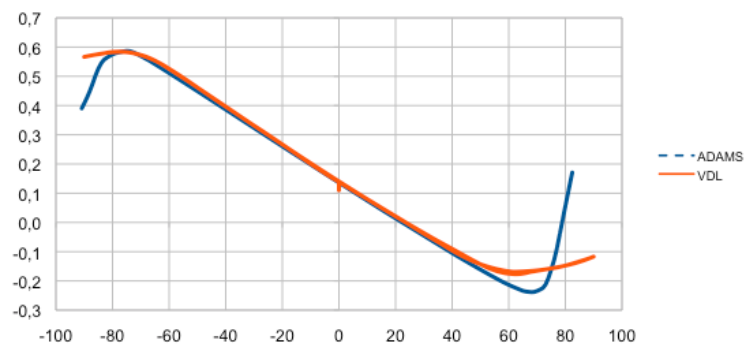

Fig. 4, Front Axle K\&C-Test: Toe Angle (degree) vs. Wheel Travel (mm)

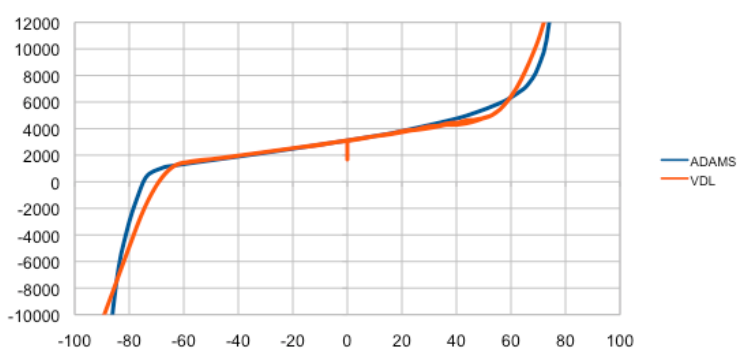

Fig. 5, Front Axle K\&C-Test: Vertical Force (N) vs. Wheel Travel (mm)

In summary the $K \& C$ results achieved the expected accordance between VDL and ADAMS or have explainable deviance due to differences in modelling. The quality of $\mathrm{K} \& \mathrm{C}$ accordance was from a certain point on not further optimized, as not being in the focus of the project. E.g. the differences in the extreme regions of Fig. 4 and Fig. 5 are due to a different modelling approach for the bump stop.

A typical set of entire vehicle handling experiments was carried out, too. Exemplarily for the comparison an abstract of results from the fundamental experiments Step Steer Manoeuvre and Stationary Cornering are given in Fig. 6 and Fig. 7.

As tire model Pacejka's magic formula was used in both codes.

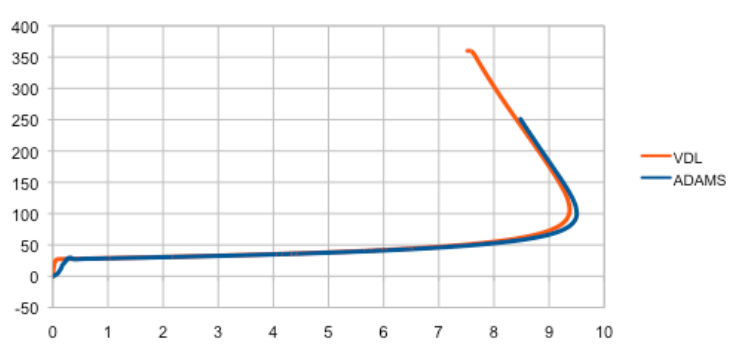

Fig. 6, Full Vehicle Steady-State Cornering: Steer Wheel Angle (degree) vs. Lat. Acceleration $\left(\mathrm{m} / \mathrm{s}^{2}\right)$

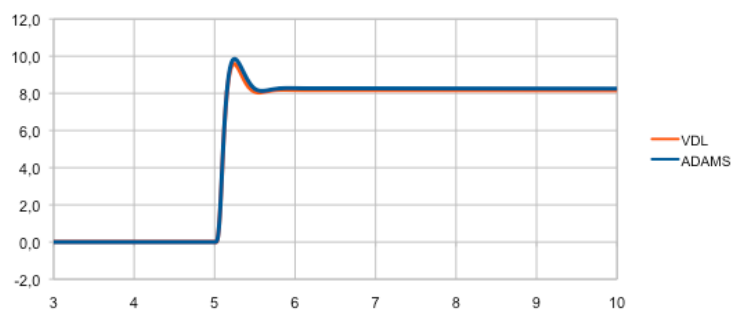

Fig. 7, Full Vehicle Step Steer: Yaw Rate (degree) vs. Time (s)

In summary the full vehicle simulations are in good accordance. It could be shown that an industry standard vehicle dynamics model in ADAMS can be redone with reasonable effort and satisfying precision within a relatively short time in a multidomain simulation tool. Having available the model there, additional non-mechanical systems can be added easily.

For the following investigations no further comparisons to the ADAMS/Car model were considered.

\section{Electric Power Steering and Controller Model}

The Electric Power Steering (EPS) model was added to the vehicle dynamics model described above in Dymola, using elements exclusively from the Modelica Standard Library. A model of the steering controller was created in Dymola, too, and alternatively the controller was added to Dymola as a Simulink model that was exported with the Real-Time Workshop using the Functional Mock-up Interface (FMI) [2]. The FMI was defined by the Modelisar consortium with the intention that dynamic system models of different software systems can be exchanged and used together for simulation. The Functional Mock-up Unit 
(FMU) essentially contains an Xml model description and a Dynamic Link Library (DLL).

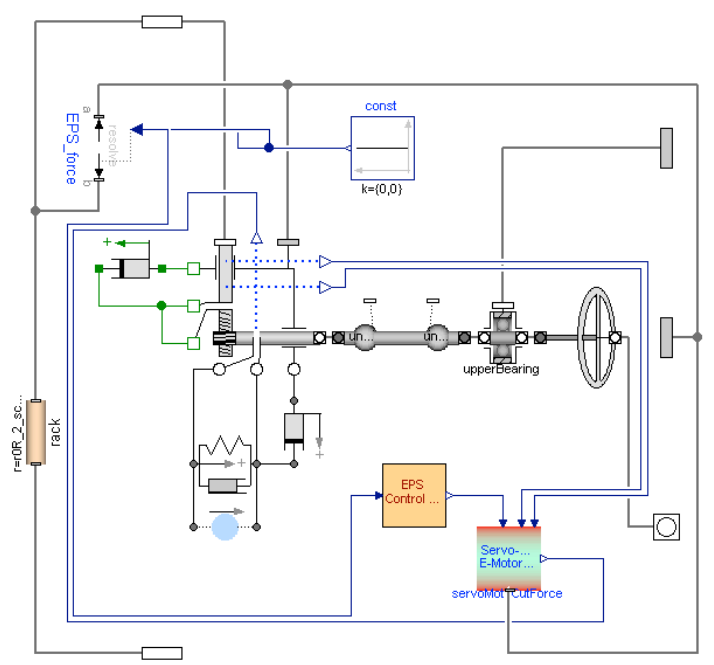

Fig. 8, Electric Power Steering and Controller Model in Dymola

The EPS model itself consists of mechanical multibody, electric and control blocks. The controller was simplified and treated as a black box of binary code, as this is the usual way that vehicle manufacturers receive the model code from their system suppliers. For this kind of pilot approach the controller was kept as simple as necessary and was designed just as a proportional gain. The output of the control unit is a drive signal for the voltage of the electric motor.

The impact of the power steering system on the dynamic vehicle behaviour was not the point of interest in this project and therefore not elaborated or tuned.

\section{Interface Concepts and accord- ing Simulation Results}

Dymola and Simulink models can be interfaced in multiple ways. For instance, the Simulink model of a controller can be imported to the Dymola model using the Functional Mock-up Interface Approach proposed by the Modelisar research project [2]. In this case Dymola serves as the solver for the entire system consisting of Modelica and Simulink subsets. Alternatively Dymola models can be exported to Simulink using e.g. the standard sfunction interface of Dymola. In this case Simulink serves as the solver.
According to the scope of the simulation work and the particular development process of the user, there are motivations for both ways. For this project, the following variants have been applied.

\begin{tabular}{|c|c|c|c|c|}
\hline & $\begin{array}{l}\text { Vehicle } \\
\text { Dynamics } \\
\text { Model }\end{array}$ & $\begin{array}{c}\text { EPS } \\
\text { Actuator } \\
\text { Model }\end{array}$ & $\begin{array}{c}\text { Steering } \\
\text { Controller } \\
\text { Model }\end{array}$ & $\begin{array}{l}\text { Simulation } \\
\text { Tool, Solver }\end{array}$ \\
\hline Variant 1 & Dymola VDL & $\begin{array}{l}\text { Dymola } \\
\text { Electric Lib }\end{array}$ & $\begin{array}{l}\text { Dymola } \\
\text { Control Lib }\end{array}$ & Dymola \\
\hline Variant 2 & Dymola VDL & $\begin{array}{l}\text { Dymola } \\
\text { Electric Lib }\end{array}$ & Simulink & Dymola \\
\hline Variant 3 & Dymola VDL & $\begin{array}{l}\text { Dymola } \\
\text { Electric Lib }\end{array}$ & Simulink & Simulink \\
\hline
\end{tabular}

Table 1, Different Interface Approaches for the Simulation of a Vehicle with EPS-System

Due to the multidomain approach of the simulation concepts described above, in any variant multiphysical results can be studied, illustrated e.g. by the analysis of the EPS motor current in Fig. 9.

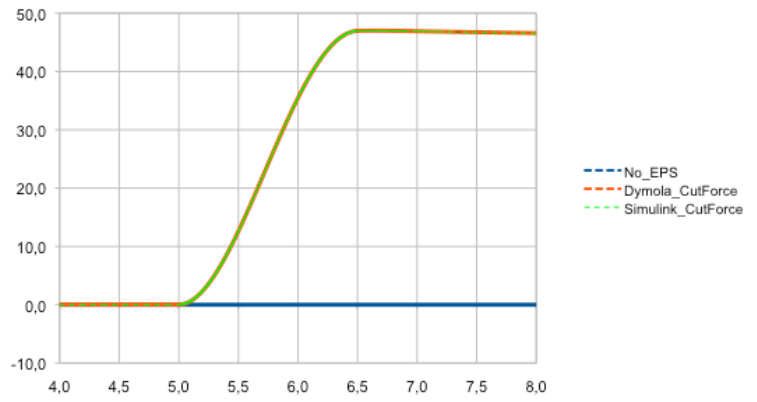

Fig. 9, Full Vehicle Step Steer: Steering Servo Motor Current (A) vs. Time (s)

Due to the different solver technologies and ways to derive equations from the system description, significant differences in the computational performance of the studied interface concepts occur for a Step Steer Manoeuvre (SSM) and Steady State Cornering Manoeuvre (SC). In all cases, however, the simulation results are practically identical. 


\begin{tabular}{llrr} 
& $\begin{array}{c}\text { Simulation } \\
\text { Tool, Solver }\end{array}$ & $\begin{array}{c}\text { Simulation } \\
\text { Time, SSM }\end{array}$ & $\begin{array}{c}\text { Simulation } \\
\text { Time, SC }\end{array}$ \\
\hline Variant 1 & Dymola & 25,2 sec. & 14,8 sec. \\
\hline Variant 2 & Dymola & $25,2 \mathrm{sec}$. & $14,8 \mathrm{sec}$. \\
\hline Variant 3 & Simulink & $25,3 \mathrm{sec}$. & $65,4 \mathrm{sec}$. \\
\hline
\end{tabular}

Table 2, Simulation Performance of Different Interface Approaches

\section{Summary and Outlook}

The work presented demonstrates that a resolved multibody model comparable to an industry standard ADAMS model can be created with reasonable effort in a multidomain simulation environment like Dymola using the Modelica approach and according specialised libraries. Extensive validation work was invested to ensure that both models lead to comparable results.

From there on it was demonstrated that entire mechatronic system simulation is easily possible in multidomain simulation tools, using vehicle dynamics, electric, additional mechanical and control models. Manifold ways to interface Dymola and Simulink support flexible approaches and tool strategies to simulate multiphysical mechatronic systems and match the particular needs of a user's specific development process.

It was shown that the described approaches have the potential to cover the needs of the upcoming challenges of e-mobility for system design.

Additional concepts to interface multidomain simulation tools like Dymola with control simulation tools like Simulink are under development at different places and promise an even tighter integration of the required tools.

\section{References}

[1] Niklas Philipson, Johan Andreasson, Magnus Gäfvert, Andrew Woodruff: Heavy Vehicles Modeling with the Vehicle Dynamics Library, Modelica 2008, March 3rd - 4th, 2008

[2] MODELISAR consortium. Functional Mock-up Interface for Model Exchange. V. 1.0.

http://www.modelisar.org, 2010 